\title{
Role of Non-Group A Streptococci in Acute Pharyngitis
}

\author{
Jeffrey Tiemstra, MD, and Rosita L. F. Miranda, MD, MS, DLO
}

Background: The role of non-group A streptococci (non-GAS) as pathogens of acute pharyngitis is controversial. Data are limited and conflicting on whether these bacteria are true pathogens of pharyngitis and whether treatment is indicated in all cases or just select cases. However, non-GAS are well-documented as being pathogens of other diseases, including neonatal sepsis, pneumonia, endocarditis, and urinary tract infections. If non-GAS are pathogens of acute pharyngitis, treatment may speed recovery as well as prevent complications. The objective of this study was to determine whether, in cases of pharyngitis in which non-GAS is identified on culture, the clinical signs and symptoms resemble those of group A streptococcal pharyngitis thus implicating them as true pathogens or if they resemble culture-negative pharyngitis, suggesting these cases are viral in etiology.

Method: This was a 3-group retrospective case-control study $(\mathrm{N}=915$; mean age, 26 years $)$. Cases included all patients with non-GAS identified on culture $(n=180)$. The control group 1 consisted of all patients with GAS infection identified by a rapid strep test or culture $(n=145)$; control group 2 included all patients with a negative rapid strep test and culture (presumed viral pharyngitis; $\mathbf{n}=584$ ). Multivariate analysis was used to compare the prevalence of 5 clinical features among the groups.

Results: The presence of headache and fever was significantly associated with streptococcal infection, with no difference between GAS and non-GAS infection. Exudates and lymphadenopathy were also significantly associated with both GAS and non-GAS infection compared with viral infection. When 2 criteria were present, the risk of any streptococcal infection rose to $55 \%$ (27\% for non-GAS or GAS); when 3 or more criteria were present, the rate of any streptococcal infection rose to 81\% (non-GAS infection, 34\%; GAS infection, $47 \%$ ).

Conclusion: In this predominantly young, adult population with acute pharyngitis, non-GAS infection was as common as GAS infection and was associated with the same clinical features typically associated with GAS. Although the benefits of treating non-GAS pharyngitis in terms of either symptomatic relief or prevention of sequelae are unproven, clinicians may want to consider treating patients with proven or presumptive non-GAS pharyngitis who fail to respond to symptomatic therapy or who are at increased risk for sequelae of group B or group C streptococcal infections, such as those patients who are or have close contact with pregnant women, neonates, and elderly or immunocompromised persons. Further study is needed to determine whether patients with non-GAS pharyngitis benefit from targeted antibiotic treatment. (J Am Board Fam Med 2009;22:663-669.)

Sore throat is one of the 5 most common reasons patients visit their primary care physicians, accounting for 11 to 18 million primary care office visits annually. ${ }^{1}$ Unfortunately, debate continues

This article was externally peer reviewed.

Submitted 25 February 2009; revised 16 June 2009; accepted 18 June 2009.

From the Family Medicine Center (JT) and the Department of Family Medicine (RLFM), University of Illinois at Chicago.

Funding: none.

Conflict of interest: none declared.

Corresponding author: Jeffrey Tiemstra, MD, Associate Professor of Clinical Family Medicine, University of Illinois at Chicago, Family Medicine Center (M/C 397), University Village, 722 West Maxwell Street, Suite 235, Chicago, IL 60607 (E-mail: jtiemstr@uic.edu). over how to evaluate and treat these patients. Guidelines agreed to by the American Academy of Family Physicians, the Centers for Disease Control and Prevention, and the American College of Physicians differ from those of the Infectious Disease Society of America (IDSA). ${ }^{2}$ Both sets of guidelines are based on the Centor clinical criteria for predicting risk of infection from group A streptococci (GAS), namely fever, absence of cough, cervical lymphadenopathy, and pharyngeal exudates. Both sets of guidelines agree that patients with 0 to 1 criteria should be neither tested nor treated and that patients with 2 to 3 criteria should be tested and treated if positive; however, the American Academy of Family Physicians/Centers for Disease 
Control and Prevention/American College of Physicians guidelines recommend empirically treating patients with 4 criteria whereas the IDSA guidelines recommend testing these patients as well and treating then only if they have a positive result. Furthermore, many physicians fail to follow any guideline and often presumptively treat patients at low risk of streptococcal infection without confirmatory testing. ${ }^{2}$

The role of non-GAS as a pathogen in acute pharyngitis is controversial. The guidelines above presume that GAS is the only important treatable cause and dissuade from using antibiotics for treating non-GAS. Studies have reported conflicting incidence rates of non-GAS in symptomatic versus asymptomatic individuals. ${ }^{3-5}$ One study of children found that group $\mathrm{C}$ streptococcus can cause exudates, sore throat, and fever, ${ }^{6}$ although whether these children respond to antibiotics remains controversial. ${ }^{7}$ Although group C pharyngitis is common in adults, only one study found rates of fever, exudates, and cervical adenopathy similar to those rates found in individuals with GAS pharyngitis. ${ }^{8}$ The consequences of untreated non-GAS pharyngitis are also unclear. Although there has been no established connection between pharyngitis and other non-GAS infections, group B and group C strep are causes of other serious diseases, including neonatal sepsis, pneumonia, endocarditis, and food-borne illness. ${ }^{9,10}$

This study examined the incidence of non-GASassociated pharyngitis among our clinical population and whether clinical criteria can be used to distinguish non-GAS from viral pharyngitis. Clinical criteria examined included the 4 Centor criteria: fever, cervical lymphadenopathy, pharyngeal exudates, and the absence of cough, as well as headache. Although headache is not one of the Centor criteria, it is a commonly looked for symptom of strep throat and is associated with group A streptococcal infection in both children and adults. ${ }^{11,12}$

\section{Methods}

We conducted a retrospective case-control study of all adult patients who presented to our 2 family medicine clinics with sore throat and had a rapid strep test (RST) during a 12-month period. Our clinics serve a mixed urban population. Approximately one third of our patient population is the student body of a large urban university. The re- mainder are primarily managed care and privately insured patients and their families, with $<10 \%$ covered by Medicare and $<10 \%$ covered by Medicaid or uninsured.

Our clinics use the QuickVue Inline Strep A test (Quidel Corp., San Diego, CA). Per our medical center policy all patients with a negative RST must have a throat culture done. Cultures are inoculated to a streptococcal-selective agar and the plates are incubated for 24 to 48 hours in $5 \% \mathrm{CO}_{2}$-enriched atmosphere at $35^{\circ}$. The Hardy Diagnostics Strep PRO Grouping Kit (Santa Maria, CA) was used for typing; this kit uses a rapid latex agglutination method for the serologic identification of Lancefield's group A, B, C, F, and G from isolated colonies of B hemolytic streptococci.

All patients with non-GAS identified on culture were included as cases. Control group 1 consisted of all patients with GAS infection identified by RST or culture; control group 2 included all patients with a negative RST and culture (presumed viral pharyngitis). Each chart was reviewed for documentation of 5 clinical criteria traditionally associated with GAS infection: headache, fever (by history or documented in the office $>99.5^{\circ}$ ), pharyngeal exudates, cervical lymphadenopathy, and absence of cough. The incidence of these criteria was compared by multivariate analysis using SPSS software (SPSS, Inc., Chicago, IL).

\section{Results}

Nine hundred fifteen charts were reviewed; 6 patients, who had negative RSTs but no cultures sent, were excluded. The mean age was 26 years for cases, 25 years for control group 1, and 27 years for control group $2(P>.05)$. The population was primarily young adults (Figure 1). The prevalence of GAS by culture or RST was $16.0 \%$, the prevalence of non-GAS was $19.8 \%$, and the presence of viral (culture negative) was $64.2 \%$ (Figure 2). Of the 145 GAS patients, RST was positive for $75 \%$ of patients; the remainder were diagnosed by culture. Prevalence rates for non-GAS by type were $8.5 \%$ (group C), 6.0\% (group B), 3.6\% (group G), 1\% (group F), and 1\% (nontypeable) (Figure 3).

The incidence of each of the clinical criteria are shown in Table 1. The presence of headache and fever was significantly associated with streptococcal infection, with no difference between GAS and non-GAS infection. Exudates and lymphadenopa- 


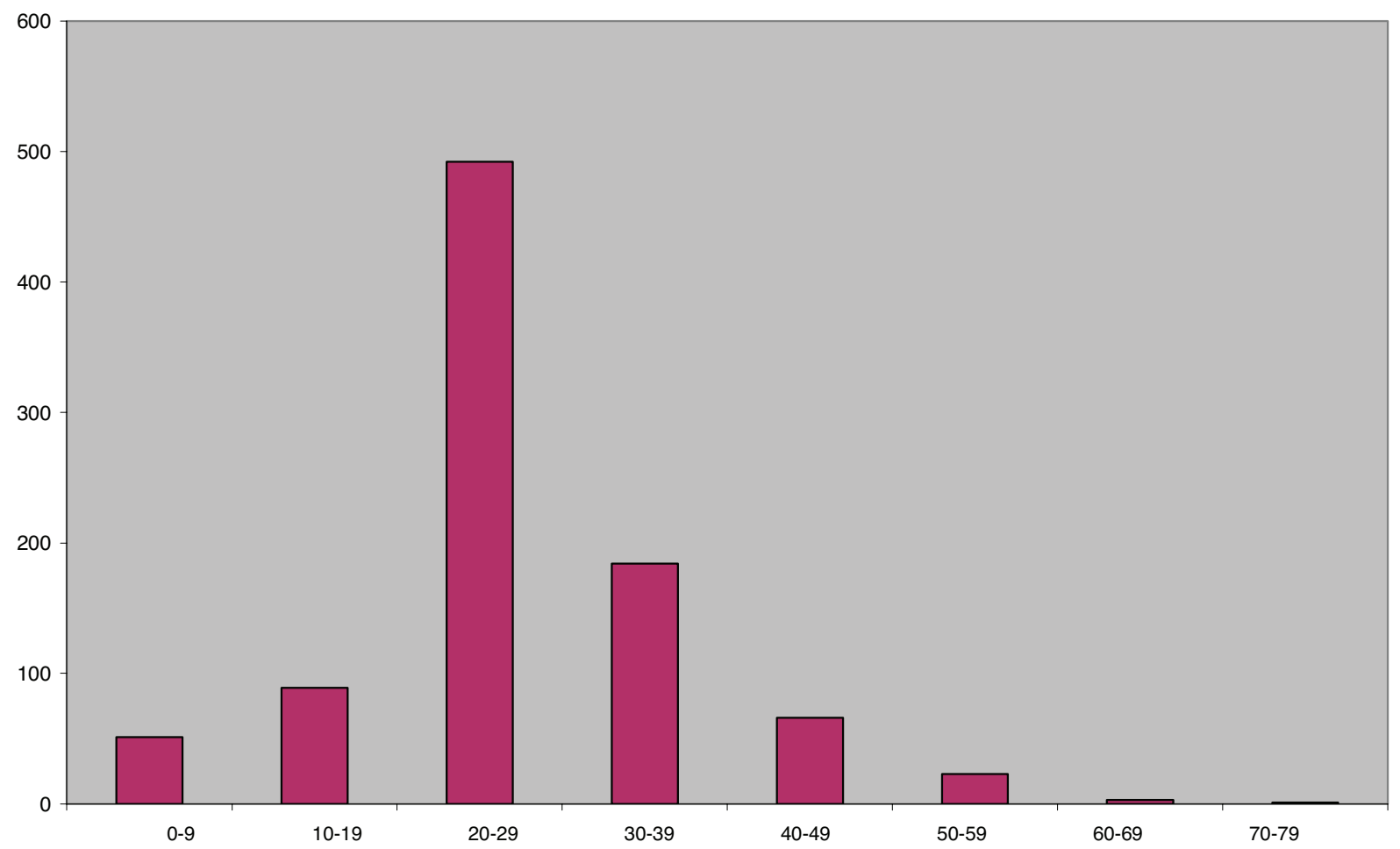

Figure 1. Age distribution of patients.

thy were also significantly associated with both GAS and non-GAS infection. Although both of these criteria were significantly more common in GAS infection than non-GAS infection, the magnitude of the difference was much smaller than between either streptococcal infection versus viral infection. The absence of cough did not differ between non-GAS infection and viral infection but was significantly more common in GAS infection (Figure 4). Analysis was also performed for individual non-GAS types. For group $\mathrm{B}$ and group $\mathrm{C}$ alone or combined the results were not significantly different; for groups F, G, and nontypeable, the numbers were too small for meaningful analysis (Figure 5).

Clinical guidelines are based on the number of criteria present; Table 2 shows the culture results by the number of criteria present for each patient. Patients with 0 or 1 criteria had an $85 \%$ and $72 \%$

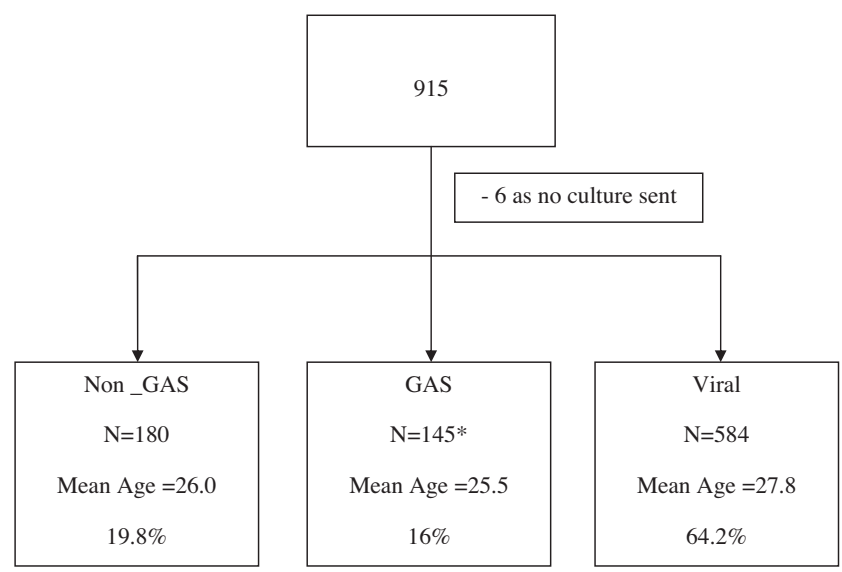

Figure 2. Study flow diagram. $* 75 \%$ by rapid strep test and $25 \%$ by culture. GAS, group A streptococci. 


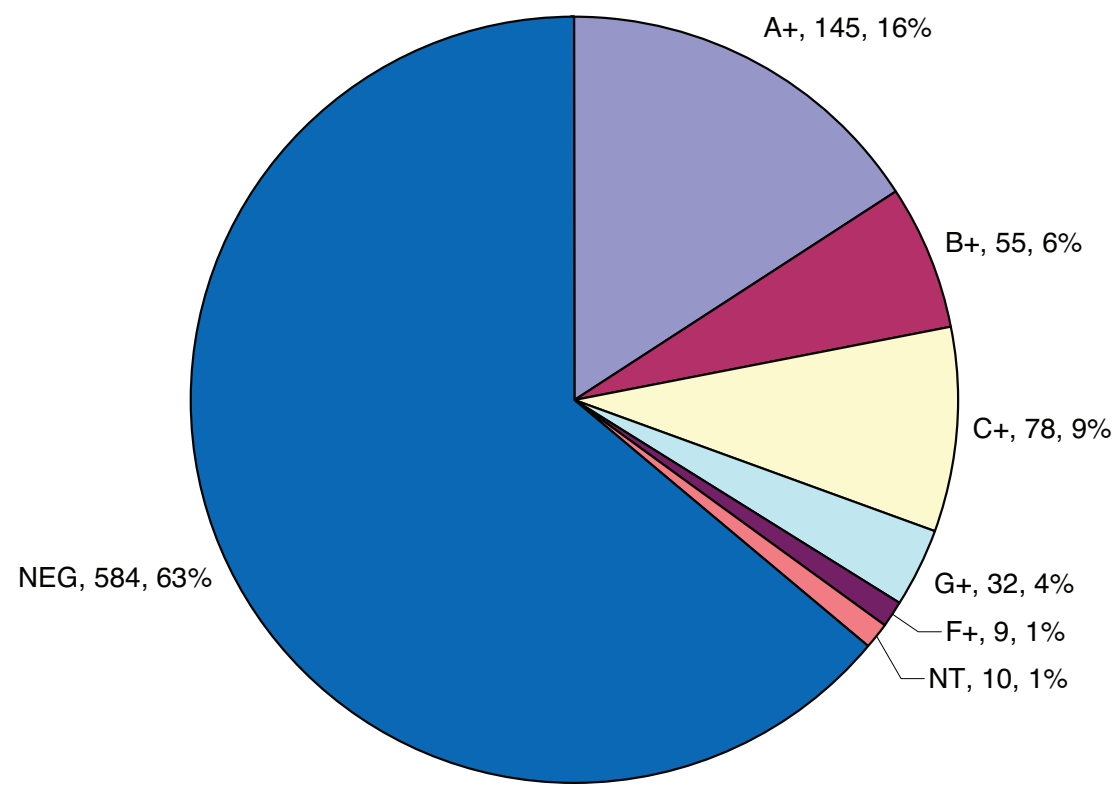

Figure 3. Prevalence rates by type of streptococci.

rate of viral infection, respectively. When 2 criteria were present, the risk of any streptococcal infection rose to $55 \%$ (27\% for non-GAS or GAS), and when 3 or more criteria were present the rate of any streptococcal infection rose to $81 \%$ (non-GAS infection, 34\%; GAS infection, 47\%; Figure 6). The frequencies of these clinical features for patients with GAS is consistent with frequencies reported in other studies.

\section{Discussion}

As a retrospective study we could not ensure capture of all patients who presented to the clinics with a chief complaint of sore throat during this time frame. Billing code data were reviewed but ultimately this was not useful because patients with negative tests could be billed under multiple diagnoses, such as upper respiratory infection, cough, or viral syndrome. However, because our rates of GAS infection were similar to those reported in other studies, and because 589 patients tested had only 0 or 1 criteria present, it seems likely that we captured a representative majority of patients presenting with sore throat. It is possible that some patients with 0 or 1 criteria were not tested, but it is also possible that patients with 4 or 5 criteria were presumptively treated without testing. Given these factors it seems unlikely that missed patients would skew the results significantly in one direction.

In this retrospective chart review we relied on clinician documentation for the presence of clinical criteria. Clinicians may under-report clinical findings when they have a positive RST or when they are relying primarily on the RST result. Fever, exudates, and adenopathy were probably most reliably documented and notably had the strongest association with streptococcal infection. As a "negative" finding, the absence of cough was probably

Table 1. Incidence of Clinical Criteria by Pharyngitis Type

\begin{tabular}{|c|c|c|c|c|c|}
\hline Criteria & Non-GAS (\%) & GAS (\%) & $\begin{array}{c}P \\
\text { (vs Non-GAS) }\end{array}$ & Viral (\%) & $\begin{array}{c}P \\
\text { (vs Non-GAS) }\end{array}$ \\
\hline Headache & 14 & 18 & .39 & 6 & .00 \\
\hline Fever & 47 & 57 & .72 & 30 & .00 \\
\hline Exudates & 31 & 44 & .00 & 9 & .00 \\
\hline Adenopathy & 31 & 51 & .00 & 8 & .00 \\
\hline No cough & 35 & 47 & .00 & 29 & .16 \\
\hline
\end{tabular}

GAS, group A streptococci. 


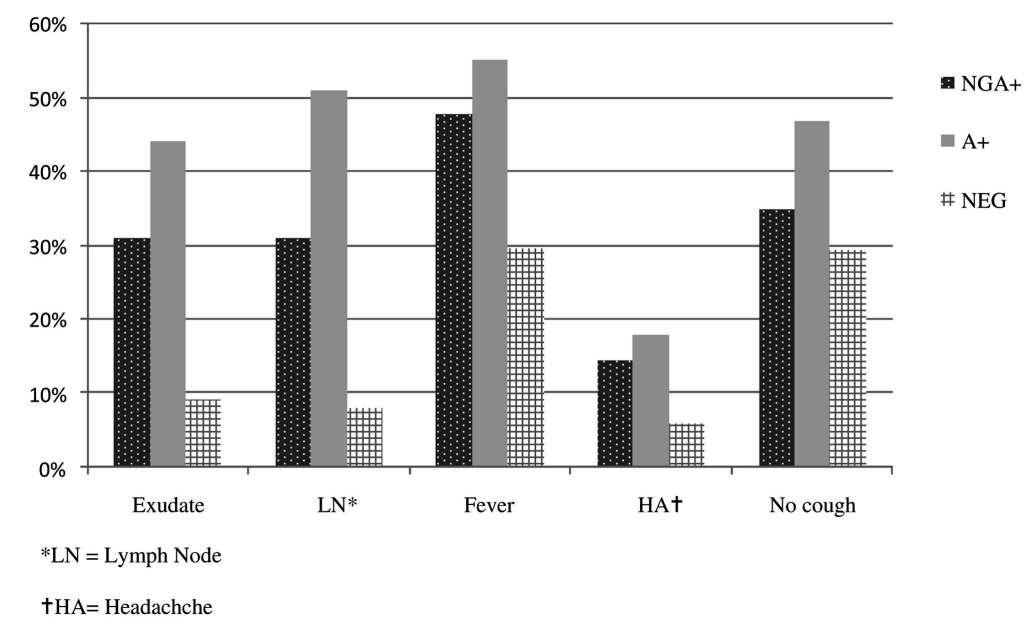

Figure 4. Comparison of the clinical features by pharyngitis type.

the least reliably documented and was found to have the weakest association with streptococcal infection. Because our clinics are teaching practices for medical students, residents, and nurse practitioner students documentation tends to be thorough. These clinics also use an electronic medical record that significantly streamlines documentation. Given the reported rates of these findings in our study un- derdocumentation does not seem to be a major confounder.

Our rapid strep test sensitivity of $75 \%$ may seem lower than expected. Although the manufacturer reports a $92 \%$ to $96 \%$ sensitivity, published studies of rapid antigen detection of GAS show rates ranging from $70 \%$ to $90 \%$, which is more consistent with our results. ${ }^{13}$

\section{Clinical Findings by Culture Result}

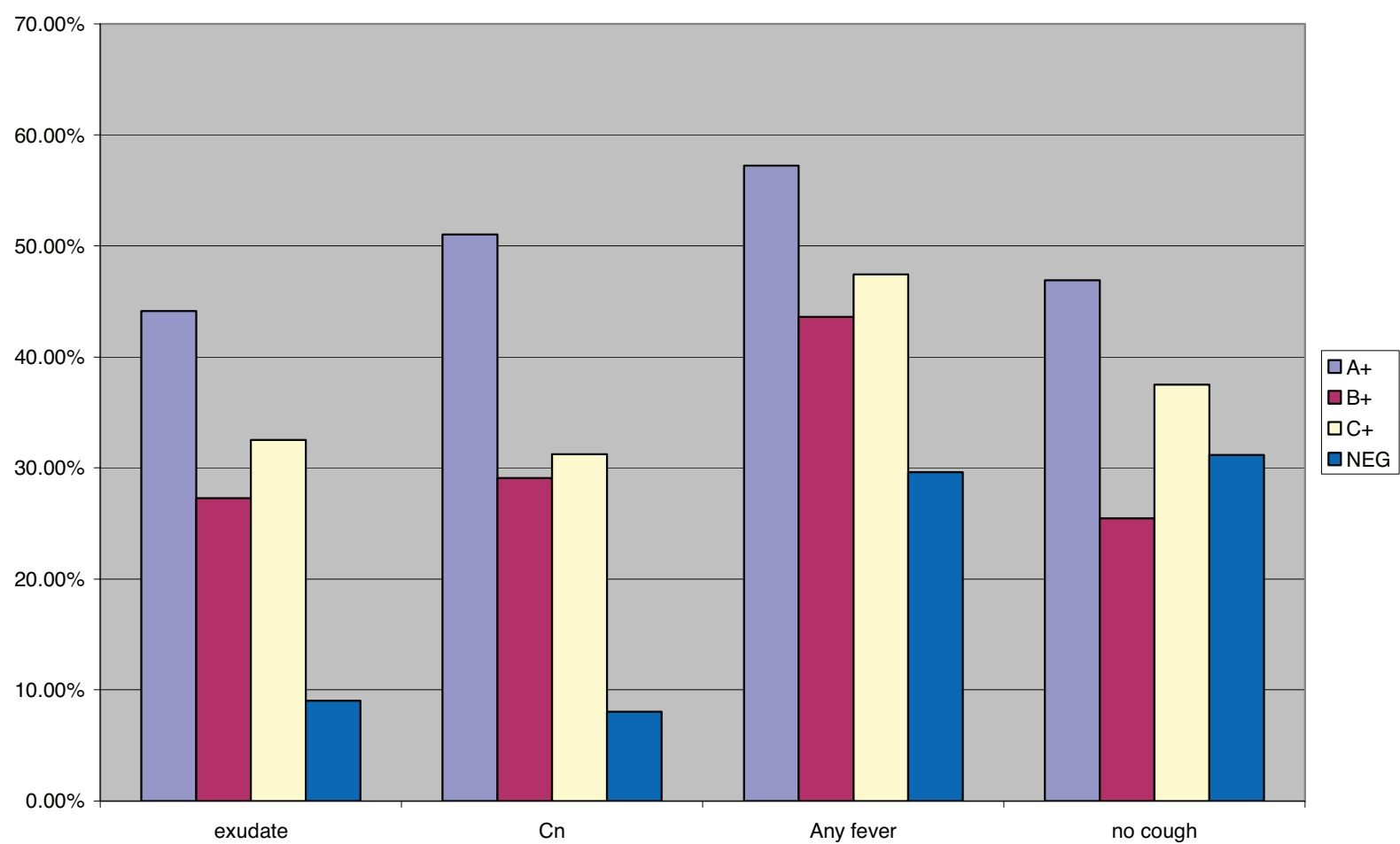

Figure 5. Clincial features for groups A, B, and C versus viral. 
Table 2. Pharyngitis Type by Number of Clinical Criteria Present

\begin{tabular}{|c|c|c|c|c|c|}
\hline Criteria (n) & GAS (n [\%]) & Non-GAS (n [\%]) & All Strep (n [\%]) & Viral (n [\%]) & Total (n) \\
\hline 0 & $10(3.7)$ & $30(11.1)$ & $40(14.8)$ & $231(85.2)$ & 271 \\
\hline 1 & $35(9.7)$ & $65(18.1)$ & $100(27.8)$ & $259(72.1)$ & 359 \\
\hline 2 & $44(27.7)$ & $44(27.7)$ & $88(55.4)$ & $71(44.7)$ & 159 \\
\hline 3 & $37(44.0)$ & $32(38.1)$ & $69(82.1)$ & $15(17.9)$ & 84 \\
\hline 4 & $17(56.7)$ & $7(23.3)$ & $24(80.0)$ & $6(20.0)$ & 30 \\
\hline 5 & $2(33.3)$ & $2(33.3)$ & $4(66.7)$ & $2(33.3)$ & 6 \\
\hline
\end{tabular}

GAS, group A streptococci.

In this study population of primarily young adults, non-GAS was as common as GAS in those with acute pharyngitis. Non-GAS infection was significantly associated with headache, fever, exudates, and cervical lymphadenopathy compared with patients a negative culture, and with frequencies approaching those of patients with GAS infection, which suggests that non-GAS are likely to be pathogens in some of these cases. The presence of 3 clinical criteria predicts non-GAS or GAS infection with equal likelihood, although the presence of 4 or 5 criteria more reliably predicts GAS infection than non-GAS infection. Although we do not have a control group of asymptomatic patients with which to compare the frequency of non-GAS isolates, the association of a symptom complex recognized as bacterial in origin is strong evidence for non-GAS as pathogens in these patients.

If the goal of treatment is to target GAS infection exclusively, then the IDSA guidelines recommending treatment for only patients who test positive seem reasonable; in this study the Centor criteria predict at best only a $50 \%$ chance of GAS infection. If the goal is to treat any streptococcal infection, then treating adult patients with 3 or more criteria is reasonable because they have an $80 \%$ chance of any streptococcal infection. At this time the benefits of treating non-GAS pharyngitis

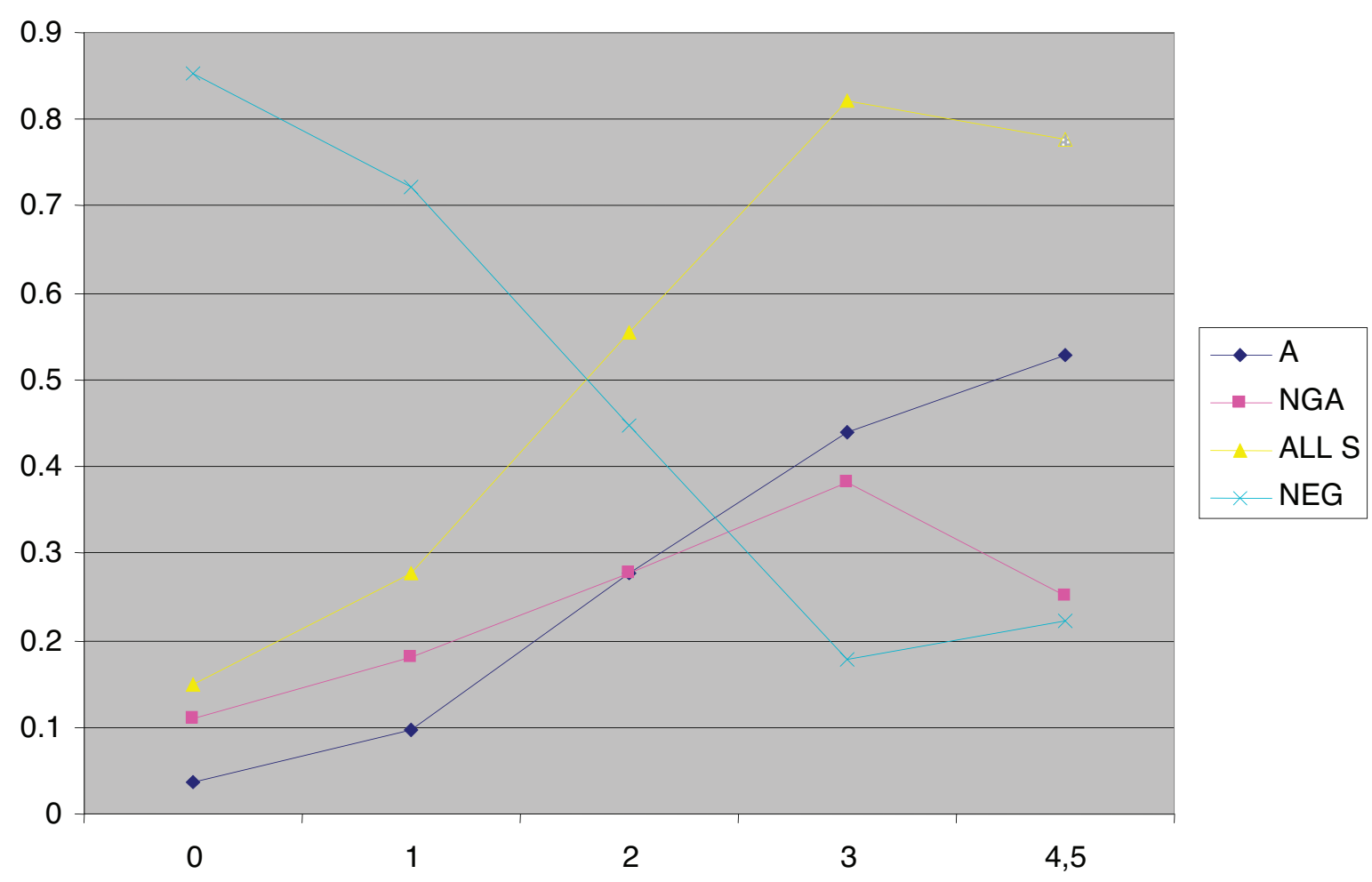

Figure 6. Pharyngitis type by the number of clinical criteria present. 
in terms of either symptomatic relief or prevention of sequelae are unproven. Clinicians may consider treating patients with proven or presumptive nonGAS pharyngitis who fail to respond to symptomatic therapy or who are at increased risk for sequelae of group B or group C streptococcal infections, such as patients who are or have close contact with pregnant women, neonates, or elderly or immunocompromised patients. Further study is needed to determine whether patients with nonGAS pharyngitis benefit from targeted antibiotic treatment.

\section{References}

1. Woodwell DA, Cherry DK. National Ambulatory Medical Care Survey: 2002 summary. Adv Data 2004;(346):1-44.

2. Linder J, Chan JC, Bates DW. Evaluation and treatment of pharyngitis in primary care practice: the difference between guidelines is largely academic. Arch Inten Med 2006;166:1374-9.

3. Cooper RJ, Hoffman JR, Bartlett JG, et al. Principles of appropriate antibiotic use for acute pharyngitis in adults. Ann Intern Med 201;134:509-17.

4. Chowdhury MNH, Kambal AM, Al-Eissa YA, Khaliq MRA, Al-Ayed IH, Al-Sanie AM. Non-group A streptococci: are they pathogens in the throat. J R Soc Health 1997;117:160-3.

5. Turner JC, Hayden FG, Lobo MC, Ramirez CE, Muren D. Epidemiologic evidence for Lancefield group $\mathrm{C}$ beta-hemolytic streptococci as a cause of exudative pharyngitis in college students. J Clin Microbiol 1997;35:1-4.

6. Benjamin JT, Perriello VA Jr. Pharyngitis due to group $\mathrm{C}$ hemolytic streptococci in children. J Pediatr 1976;89:254-6.

7. Schwartz RH, Shulman ST. Group C and group G streptococci: in-office isolation from children and adolescents with pharyngitis. Clin Pediatr 1986;25: 496-502.

8. Turner JC, Hayden GF, Kiselica D, Lohr J, Fishburne CF, Murren D. Association of group C betahemolytic streptococci with endemic pharyngitis among college students. JAMA 1990;264:2644-7.

9. Schrag SJ, Zywicki S, Farley MM, et al. Group B streptococcal disease in the era of intrapartum antibiotic prophylaxis. N Engl J Med 2000;342:15-20.

10. Centers for Disease Control (CDC). Group C streptococcal infections associated with eating homemade cheese-New Mexico. MMWR Morb Mortal Wkly Rep 1983;32:510, 515-6.

11. Chazan B, Shaabi M, Bishara E, Colodner R, Raz R. Clinical predictors of streptococcal pharyngitis in adults. Isr Med Assoc J 2003;5:413-5.

12. Lee LH, Ayoub E, Pichichero ME. Fewer symptoms occur in same-serotype recurrent streptococcal tonsillopharyngitis. Arch Otolaryngol Head Neck Surg 2000;126:1359-62.

13. Gerber MA, Shulman ST. Rapid diagnosis of pharyngitis caused by group A streptococci. Clin Microbiol Rev 2004;17:571-80. 